\title{
Competitividad de las empresas comercializadoras de materiales de construcción legalmente constituidas en la ciudad de Estelí, año 2015 $^{1}$
}

\author{
Isherly Massiel Amaya Zeas ${ }^{2}$ \\ Henry Abimael Pozo Sevilla ${ }^{3}$ \\ Francis Janireth Zelaya Obando ${ }^{4}$ \\ Beverly Castillo Herrera ${ }^{5}$
}

\section{RESUMEN}

En el presente estudio se analizaron las estrategias que utilizan las empresas comercializadoras de materiales de construcción para ser competitivas en el mercado Esteliano. Según el nivel de conocimiento es una investigación aplicada y según el enfoque filosófico es una investigación cuantitativa. El universo de estudio lo constituyen las empresas comercializadoras de materiales de construcción. Las técnicas de recolección de datos son encuestas y entrevistas las cuales se aplicaron a los propietarios o encargados de las empresas, además se aplicaron encuestas a los clientes. En los principales resultados se muestra que las estrategias más utilizadas por las empresas son ubicación, precios bajos y con mayor importancia la atención brindada a los clientes.

Palabras claves: Competitividad, Comercialización, Materiales de construcción, Estrategia, empresa.

Recibido: 10 de diciembre de 2015

Aceptado: 12 de enero de 2016

1 Este artículo se elabora a partir de la tesis titulada Competitividad de las empresas comercializadoras de materiales de construcción legalmente constituidas en la ciudad de Estelí, año 2015. Para optar al título de administración de empresas en el año 2015 por la UNAN- MANAGUA, FAREM-ESTELI

2 UNAN-Managua, FAREM-Estelí. Correo Electrónico: zamyamaia@gmail.com

3 UNAN-Managua, FAREM-Estelí. Correo Electrónico: henryabimael12@gmail.com

4 UNAN-Managua, FAREM-Estelí. Correo Electrónico: Janyzelaya@gmail.com

5 Candidata a doctora en Ciencias Sociales por la Universidad del Zulia. Maestría en Ciencias Sociales por la Universidad de Guadalajara. Docente titular de la UNAN-Managua, FAREM-Estelí. Correo Electrónico: beverly. castillo@yahoo.com 


\section{Competitiveness of companies selling construction materials legally established in the city of Esteli, in 2015}

\section{SUMARY}

In this study the strategies used by the traders of construction materials to be competitive in the Estelian market, were analyzed. This is an applied and quantitative research. The universe of study are the trader companies of building materials. The data collection techniques are surveys and interviews. They were applied to the owners or managers of companies, the surveys were applied to customers. The main results show that the strategies most used by these companies are their location, low prices and more importantly the service provided to their clients.

Keywords: Competitiveness, Marketing, Building Materials, Strategy, company 


\section{INTRODUCCIÓN}

"La industria de la construcción en Nicaragua experimento un crecimiento de $2.8 \%$ y representó el $4.8 \%$ del Producto Interno Bruto en el año 2014, contribuyendo así en un $0.1 \%$ a la tasa de crecimiento del PIB”. Actualmente la ciudad de Estelí cuenta con 15 empresas comercializadoras de materiales de construcción legalmente constituidas de las cuales podemos destacar: SINSA, El Halcón, Construnica, El constructor, entre otras. La competitividad es importante ya que les permite desarrollarse y posicionarse en la industria de la construcción, sin embargo existen ciertas limitantes que impiden que se mantengan en el mercado, uno de estas es la falta de publicidad, debido a los elevados costos que representa el uso de este instrumento.

Otro factor que afecta negativamente a las empresas es la ubicación geográfica, principalmente a las que se sitúan en las calles centrales de la ciudad, debido a que no cuentan con área de parqueo lo que dificulta la compra y movilización de los materiales y de igual forma afecta la circulación de otros vehículos.

Debido al crecimiento económico del departamento de Estelí, han surgido diversas empresas comercializadoras de materiales de construcción lo que ha provocado la disminución de las ventas en las empresas que poseen años en el mercado.

En muchas ocasiones los propietarios implementan estrategias competitivas con el objetivo de captar un mayor número de clientes, sin embargo dichas estrategias no han dado el resultado esperado o no se han implementado a como se planificaron, por ende la afluencia de clientes ha sido mínima, ya que no se sienten completamente satisfechos por el servicio recibido.

Los gerentes o encargados no son capaces de reconocer sus debilidades, ni de corregirlas lo cual evita el crecimiento de la empresa y el de ellos como empresarios. En muchos casos presentan adversidad al cambio, lo cual no permite la implementación de nuevas estrategias que aceleren los procesos administrativos y que aumenten las utilidades.

Para toda empresa es fundamental conocer su mercado y los cambios que pueda experimentar, además es importante identificar las fortalezas y debilidades que poseen como empresa para mejorar su capacidad competitiva. Los propietarios deben tomar decisiones inmediatas en cuanto a Innovación y generación de valor en sus productos, así podrán garantizar la supervivencia y viabilidad de sus empresas en el mercado de los materiales de construcción, sin embargo los propietarios desconocen ciertos aspectos relacionados a la competitividad que podrían maximizar sus ventas.

Este estudio será de utilidad para las empresas comercializadoras de materiales de construcción por que les permitirá conocer su competencia: toma de decisiones, estrategias utilizadas, atención al cliente, entre otros aspectos que les permitirán identificar los factores a mejorar.

Además les permitirá disponer de mayores conocimientos de su posicionamiento en el mercado y que aspectos se deben mejorar para incrementar las ventas, por medio de la implementación de estrategias el desarrollo y crecimiento de las empresas podría optimizarse.

Así mismo será de mucha ayuda e importancia para los estudiantes de los próximos años, al momento de realizar investigaciones de temas similares ya que les permitirá tener mayores conocimientos del impacto económico que han tenido las empresas en la población Esteliana.

El Objetivo principal del estudio es conocer las estrategias empresariales implementadas por las 
empresas dedicadas a la comercialización de materiales de construcción en la ciudad de Estelí, año 2015. Lo que facilitara la propuesta de nuevas estrategias para mejorar el funcionamiento de las empresas.

\section{Los principales ejes teóricos del estudio son:}

Variedad de productos: Se refiere a los diferentes diseños o tipos de productos fabricados en una planta. Productos distintos, tanto en forma como en tamaño, desempeñan funciones diferentes y se destinan a diferentes mercados. (Groover, M. (2002). Pág. 21)

Satisfacción del cliente: Es un tipo de respuesta o evaluación positiva que hace el cliente sobre el consumo o uso de un bien o servicio cubriendo sus necesidades y expectativas. (Kotler, P. 2003. Pág. 10) Competitividad: indica la capacidad que tiene una empresa, o un territorio de producir determinados bienes y/o servicios para su mercado. La cual depende de la administración de los recursos disponibles. (Gómez, M. (2005).

\section{MATERIALES Y METODOS}

El universo de la investigación está conformado por las empresas dedicadas a la comercialización de materiales de construcción ubicados en la ciudad de Estelí.

En la Alcaldía Municipal de Estelí, existen 15 empresas comercializadoras de materiales de construcción y 53 ferreterías legalmente inscritas de las cuales 15 también comercializan materiales de construcción. Siendo el Universo de estudio los 30 negocios que se dedican a la comercialización de materiales de construcción legalmente inscritos.

El trabajo de campo se realizó en diversos puntos de la ciudad de Estelí, donde se aplicaron 30 encuestas a los propietarios o encargados de las empresas comercializadoras de materiales de construcción; se visitaron las instalaciones de las empresas donde se obtuvieron las estrategias competitivas que emplean los negocios, además se realizaron encuestas dirigidas a los clientes de las empresas que se consideran más competitivas, para valorar la satisfacción de los clientes.

En algunos casos ya no existían o solo eran ferreterías por lo que se decidió visitarlas a todas. Por falta de disposición y falta de tiempo por parte de los gerentes y propietarios de dos ferreterías. Se decidió sustituirlas por la Ladrillería Lina, Ladrillería Rufino González y La impulsadora del Norte quienes amablemente nos brindaron su atención.

Según la información recopilada en el trabajo de campo se procedió a la tabulación de los resultados, lo cual se realizó de forma electrónica a través del programa Word y Excel, luego se establecieron las conclusiones del estudio una vez finalizado el proceso.

\section{RESULTADOS Y DISCUSIÓN}

A través del estudio de campo se obtuvieron datos que permitieron analizar la información brindada por parte de los encuestados; se determinó que un $27 \%$ de los propietarios de las empresas comercializadoras de materiales de construcción oscilan entre las edades de 30 a 35 años, lo cual indica que forman parte de la población económicamente activa de la ciudad de Estelí, por otra parte un 13\% de los encuestados tienen entre 46 a 50 años de edad considerando que la experiencia y los conocimientos adquiridos les ha permitido posicionarse y seguir en el mercado local. 
Cuadro $N^{\circ} 1$ Edad de los propietarios

\begin{tabular}{lll}
\hline \multicolumn{3}{c}{ Universo de estudio 30 encuestas } \\
\hline \multicolumn{1}{c}{ Rango Edad } & Frecuencia & \% \\
\hline $30-35$ & 8 & $27 \%$ \\
$36-40$ & 7 & $23 \%$ \\
$41-45$ & 5 & $17 \%$ \\
$46-50$ & 4 & $13 \%$ \\
$51-$ Más & 6 & $20 \%$ \\
Total & 30 & $100 \%$ \\
\hline
\end{tabular}

Fuente: Datos primarios de la encuesta. Junio 2015

Tomar decisiones es de suma importancia dado que esto afecta positiva o negativamente la empresa, a lo cual el 50\% de los propietarios afirmaron ser ellos los que toman las decisiones de las inversiones que van a realizar, en que tiempo y forma hacerlo, además son los que tienen la última palabra para elegir que estrategias implementar para mejorar su competitividad.

\section{Cuadro $\mathrm{N}^{\circ} 2$ Toma de decisiones}

\begin{tabular}{lcc}
\hline \multicolumn{3}{c}{ Universo de estudio 30 encuestas } \\
\hline \multicolumn{1}{c}{ Decisiones } & Frecuencia & \% \\
\hline Propietario & 15 & $50 \%$ \\
Administrador & 9 & $30 \%$ \\
Otro & 6 & $20 \%$ \\
Total & 30 & $100 \%$ \\
\hline
\end{tabular}

Fuente: Datos primarios de la encuesta. Junio 2015

Por otra parte un $20 \%$ de los propietarios asumen no ser directamente ellos los que toman las decisiones en algunos casos son: los encargados, familiares u otros, sin embargo en algunos casos las decisiones más importantes son tomadas por los socios quienes realizan reuniones y analizan las posibles soluciones, en otros casos algunos de ellos realizan consultas a un asesor externo sobre qué decisión podría tomar o cual le convendría más.

Es de gran importancia conocer qué fortalezas les han permitido mantenerse en el mercado, a la cual respondieron con un $90 \%$ que consideran su principal fortaleza la atención al cliente ya que para ellos lo primordial son los clientes y se les debe de tratar con respeto y amabilidad debido a que son la base de su economía, sin embargo un $67 \%$ consideran que la variedad de productos es su mayor fortaleza debido a que garantizan los productos que los clientes más demandan, algunos dueños mencionaron que es esencial contar con todos los productos que se necesitan en una construcción, lo que les ha generado un aumentado en sus ventas por lo que los clientes se sienten satisfechos al adquirir todos sus productos en un mismo lugar.

Cuadro $N^{\circ} 3$ Fortalezas que permiten el éxito de la empresa

\begin{tabular}{llc}
\hline \multicolumn{3}{c}{ Universo de estudio 30 encuestas } \\
\hline Fortaleza & Frecuencia & \% \\
\hline Atención al Cliente & 27 & $90 \%$ \\
Precios & 18 & $60 \%$ \\
Materiales de Calidad & 15 & $50 \%$ \\
Publicidad & 11 & $37 \%$ \\
\hline Diversificación de Productos & 20 & $67 \%$ \\
\hline
\end{tabular}

Fuente: Datos primarios de la encuesta. Junio 2015

Un $60 \%$ considera que los precios que les brindan a sus clientes ya que la mayoría busca precios accesibles. En cambio un $37 \%$ afirmo que además de las fortalezas mencionadas anteriormente, consideran que la publicidad es uno de sus potenciales porque les permite llegar a clientes que se encuentren en los departamentos o en otras ciudades del país y así tienen conocimiento de la empresa y sus materiales. El Gerente de SINSA explica:

"Estar comprometidos con la labor que realizamos, brindar una mejor atención a nuestros clientes, disponer de valores dentro de la empresa que los hacemos cumplir día a día, mejorar como asesores de venta, cajero, vendedor, evitar errores en el proceso y cumplir con nuestros objetivos; son aspectos que han llevado a tener éxito en el mercado local y que permiten que la organización crezca."(Gerente de SINSA) 
Los propietarios han realizado alguna mejora en los últimos años en la empresa a lo que un $77 \%$, expreso que han decidido ofrecer una gama más amplia de productos para que los clientes adquieran en un mismo lugar todo lo que necesitan y se sientan más satisfechos con sus compras, en cambio el $47 \%$ menciona haber ampliado sus instalaciones lo que permite disponer de un mayor espacio para los materiales y les permite ingresar más productos, además los clientes cuentan con un lugar más amplio para seleccionar los materiales.

\begin{tabular}{lll}
\multicolumn{3}{c}{ Cuadro $\mathbf{N}^{\circ} \mathbf{4}$ Mejoras en los últimos años } \\
\hline \multicolumn{3}{c}{ Universo de estudio 30 encuestas } \\
\hline \multicolumn{1}{c}{ Mejoras } & Frecuencia & $\%$ \\
\hline Ampliar Instalaciones & 14 & $47 \%$ \\
Establecer Sucursales & 9 & $30 \%$ \\
Diversificación de productos & 23 & $77 \%$ \\
Otros & 4 & $13 \%$ \\
\hline
\end{tabular}

Fuente: Datos primarios de la encuesta. Junio 2015

El otro $13 \%$ de los propietarios realizaron otras mejoras con el propósito de renovar su empresa y el servicio que ofrece el personal, entre estas se encuentra la implementación de la publicidad y capacitación para el personal. A lo que la Gerente de SINSA explica:

"Los vendedores son capacitados en el proceso de inducción, durante un mes a cerca de productos, precios y además se les proporciona constantemente documentación audiovisual, folletos, investigan nuevas tendencias del mercado, en muchas ocasiones surgen nuevos productos por ello se les debe capacitar sobre qué materiales pueden utilizar para colocar la cerámica por ejemplo, ya que no toda se aplica solo con cemento y arena en algunos casos necesita de otros componentes." (Gerente de SINSA)

Cada empresa debe de contar con estrategias de crecimiento para atraer una mayor cantidad de clientes potenciales o mantener a sus clientes permanentes, según el universo de estudio un $7 \%$ de los propietarios consideran que disponer de precios bajos es una de las estrategias que les ha permitido desarrollándose comercialmente y ser más visitado por sus clientes. El 50\% de los propietarios reconocen que disponer de una mayor diversidad de productos les ha permitido tener un mayor crecimiento y reconocimiento dentro del mercado local; un $40 \%$ consideran que mejorar la calidad del servicio que ofrecen a sus clientes es de gran importancia para poder posicionarse, ser más demandados y reconocidos.

Cuadro $\mathrm{N}^{\circ} 5$ Estrategia de Crecimiento

\begin{tabular}{lll}
\hline \multicolumn{3}{c}{ Universo de estudio 30 encuestas } \\
\hline Estrategia de crecimiento & Frecuencia & \% \\
\hline Innovación & 7 & $23 \%$ \\
Diversificación de productos & 15 & $50 \%$ \\
Expansión territorial & 5 & $17 \%$ \\
Efectividad comercial & 6 & $20 \%$ \\
Calidad de servicio & 12 & $40 \%$ \\
Canales de distribución & 6 & $20 \%$ \\
Alianzas estratégicas & 8 & $27 \%$ \\
Otros (Especifique) & 2 & $7 \%$ \\
\hline
\end{tabular}

Fuente: Datos primarios de la encuesta. Junio 2015

E1 27\% considera que disponer de alianzas estratégicas con empresas constructoras y con albañiles locales les ha permitido ser más reconocidos y aceptados por la población al momento de comprar los diferentes materiales para la construcción de viviendas o edificios; por otra parte el $23 \%$ considera que ofrecen productos de mayor calidad los que le permitirá disponer de un mayor preferencia por los clientes y a su vez estar mejor posicionados en el mercado local. El Gerente de Ferromax explica:

"Nuestras estrategias están enfocadas en el mercado, más que todo nos basamos en la calidad de nuestros materiales, en la diferenciación del material que nosotros ofrecemos, además procuramos conocer el mercado debido a que es la base de nuestra economía. Un aspecto importante es conocer las ventajas y desventajas que posee el producto para enfatizar más en la ventajas que sería el valor del producto no tanto el precio; porque el valor es aquello intangible que te va a beneficiar y el precio es algo que se paga en 
el momento, el precio podría ser bajo o podría ser alto. De qué sirve que sea bajo si no posee la calidad suficiente y luego se deba invertir más." (Gerente Harvin Castillo, Ferromax)

La utilización de estas estrategias de crecimiento les ha traído beneficios a las empresas comercializadoras de materiales de construcción lo que les han permitido posicionarse en el mercado local con un 50\%; mientras tanto un $43 \%$ consideran que realizar cambio en sus estrategias les ha permitido disponer de una mayor demanda por parte de los clientes al momento de realizar sus compras en el local.

\section{Cuadro $\mathrm{N}^{\circ} 6$ Ventajas Competitivas}

\begin{tabular}{llc}
\hline \multicolumn{3}{c}{ Universo de estudio 30 encuestas } \\
\hline \multicolumn{1}{c}{ Ventajas competitivas } & Frecuencia & \% \\
\hline Posicionamiento en el mercado & 15 & $50 \%$ \\
Incrementó de Utilidades & 8 & $27 \%$ \\
Mayor demanda & 13 & $43 \%$ \\
Otros (Especifique) & 4 & $13 \%$ \\
\hline
\end{tabular}

Fuente: Datos primarios de la encuesta. Junio 2015

Mientras tanto un $13 \%$ de los encargados consideran que gracias a ellas disponen de una mayor preferencia por que han decidido ofrecerles algún tipo de incentivo a los clientes permanentes e implementar diferentes estrategias que les permitan captar un mayor número de clientes.

El 67\% de los propietarios consideran que deben de realizar mejoras en sus empresas en cuanto a disponer de una mayor variedad de productos y contar con el servicio de entregas a domicilio les permitiría tener una mayor fidelidad de sus clientes ya que la mayoría de las personas prefieren que les ofrezcan este servicio para evitar que los materiales sufran algún daño.
Cuadro $N^{\circ} 7$ Mejoras del servicio en el negocio

\begin{tabular}{lll}
\hline \multicolumn{3}{c}{ Universo de estudio 30 encuestas } \\
\hline \multicolumn{1}{c}{ Servicio en el negocio } & Frecuencia & \% \\
\hline Entrega a domicilio & 20 & $67 \%$ \\
Precios & 12 & $40 \%$ \\
Atención al cliente & 9 & $30 \%$ \\
Variedad de productos & 20 & $67 \%$ \\
Otros (Especifique) & 8 & $27 \%$ \\
\hline
\end{tabular}

Fuente: Datos primarios de la encuesta. Junio 2015

El $40 \%$ consideran que al disponer de precios más bajos, los clientes se ven más atraídos a adquirir los productos en sus establecimientos debido a que estos pueden optar por comprar una mayor cantidad. Mientras el $30 \%$ consideran que deben de realizar mejoras en la atención que se les brinda a los clientes debido a que esto les permite dejar una buena impresión a los clientes además hará que los clientes regresen a sus empresas, solo un $27 \%$ han considerado que deben de realizar ampliaciones de sus locales para poder ofrecer una mayor variedad de productos para satisfacer las demandas de la población. A lo que la Gerente de SINSA explica:

"Ofrecer una mayor variedad de productos, categorías, calidad y precio es de gran importancia para la empresa; porque podemos encontrarnos con un cliente que tenga la necesidad pero tal vez su capacidad de pago sea menor entonces podrías ofrecerle un material que cumpla con su necesidad y pueda pagar él a un precio más bajo; también en el caso de las variedades de productos se refiere a que el cliente no solamente adquiera los productos básicos para realizar su construcción, si no que la empresa cuente con todos los accesorios para lo que vaya a realizar". (Gerente de SINSA).

\section{Según los clientes}

El $40 \%$ de las personas encuestadas sus edades oscilan entre 31 a 40 años y son los que visitan más las empresas, debido a la experiencia adquirida en 
la adquisición de los materiales de construcción. Mientras que un $25 \%$ oscilan entre las edades de 41 50 años siendo clientes que realizan compras casuales ya sea para ampliaciones o remodelaciones de sus hogares o negocios propios. Por otro lado un $12 \%$ son jóvenes entre las edades de 20 a 30 años los cuales están incursionando en el mercado de construcción, o bien comenzando a construir sus hogares.

Cuadro $\mathrm{N}^{\circ} 8$ Edad de los encuestados

\begin{tabular}{|c|c|c|}
\hline \multicolumn{3}{|c|}{ Universo de estudio 481 encuestas } \\
\hline Edad & Frecuencia & $\%$ \\
\hline $20-30$ & 60 & $12 \%$ \\
\hline $31-40$ & 192 & $40 \%$ \\
\hline $41-50$ & 119 & $25 \%$ \\
\hline $51-60$ & 110 & $23 \%$ \\
\hline Total & 481 & $100 \%$ \\
\hline
\end{tabular}

Fuente: Datos primarios de la encuesta. Junio 2015

Según los datos obtenidos mediante el estudio se determinó que la principal empresa que cumple con las expectativas de los clientes es SINSA con el $77 \%$ los cuales comentaron que esta empresa ofrece gran variedad de materiales para la construcción o para la remodelación de sus hogares, posee también diferentes estilos de cerámica, azulejos, entre otros, sus instalaciones son amplias, y brindan asesorías al momento de adquirir los materiales. Por otra parte con el $46 \%$ se encuentra Construnica ya que además de contar con variedad de productos brindan buena atención e incentivos a sus clientes. Mientras tanto La Popular y El Halcón con el 40\% los encuestados dijeron visitar estas empresas por la atención y los precios ya que son favorables. A lo que una clienta agrego:

"En muchas ocasiones que decido hacer alguna mejora o construir en mi casa pienso en SINSA, ya que normalmente encuentro todo lo que necesito y aunque los precios normalmente son altos los productos son de calidad por lo recompensa de esa forma su costo" (Magdalena Lagos)

\section{Cuadro $N^{\circ} 9$ Empresas que cumplen con sus expectativas}

\begin{tabular}{lcc}
\hline \multicolumn{3}{c}{ Universo de estudio 481 encuestas } \\
\hline \multicolumn{1}{c}{ Empresas } & Frecuencia & \% \\
\hline SINSA & 369 & $77 \%$ \\
Construnica & 221 & $46 \%$ \\
La Popular & 193 & $40 \%$ \\
El Halcón & 193 & $40 \%$ \\
EPCA & 180 & $37 \%$ \\
Metamacon & 140 & $29 \%$ \\
\hline
\end{tabular}

Fuente: Datos primarios de la encuesta. Junio 2015

Los principales aspectos por los que los clientes prefieren estas empresas representado con el 58\% es porque disponen de gran variedad de productos ya que esto les permite a los clientes realizar sus compras en una misma empresa economizando tiempo y transporte, otro factor que toman en cuenta es que algunas de estas empresas cuentan con el servicio de entregas a domicilio reflejado con el $37 \%$ del total encuestado este factor representa un ahorro al cliente que no tendrá que buscar medios para trasportar sus materiales.

Cuadro $N^{\circ} 10$ Expectativas de los clientes

\begin{tabular}{lcc}
\hline \multicolumn{3}{c}{ Universo de estudio 481 encuestas } \\
\hline \multicolumn{1}{c}{ Expectativas } & Frecuencia & \% \\
\hline Variedad de productos & 280 & $58 \%$ \\
Hacen rebajas & 212 & $44 \%$ \\
Bajos precios & 190 & $40 \%$ \\
Entregan a domicilio & 180 & $37 \%$ \\
Otros & 143 & $30 \%$ \\
\hline
\end{tabular}

Fuente: Datos primarios de la encuesta. Junio 2015

Mientras que el 30\% menciono que otro aspecto que toman en cuenta es la ubicación ya que la mayoría de estas se localizan a orillas de la carretera panamericana y en el centro de la ciudad debido a que en algunas ocasiones los materiales requieren de transporte a otros municipios y esto les permite disponer de medios más accesibles para el trasporte de sus materiales tales como buses o acarreos cercanos. 
La mayor parte de los encuestados representado por el $71 \%$ considera que la atención brindada por estas empresas al momento de realizar sus compras es muy buena por lo que se sienten satisfechos; debido a que los vendedores son amables y en su mayoría ellos les brindan asesoría para que los clientes que desconocen o tienen poco conocimiento sobre el material que desean adquirir. El 1\% restante considera que la atención brindada por los vendedores es mala ya que en algunas ocasiones no les brindan información de los materiales que demanda, además comentaron que no tenían la paciencia para atenderles. A lo que un cliente dijo:

"Para mí como comprador es indispensable que me sugieran que producto puedo utilizar de no encontrarse el que busco, o que me expliquen cómo utilizar un nuevo producto, por ejemplo la cerámica necesita de muchos cuidados para que no se dañe al pegarla en el suelo y según me dijeron para que dure más se utilizan otros materiales adicionales.” (Don Antonio Suarez)

Cuadro $N^{\circ} 11$ Atención de las empresas

\begin{tabular}{lll}
\hline \multicolumn{3}{c}{ Universo de estudio 481 encuestas } \\
\hline \multicolumn{1}{c}{ Valoración } & \multicolumn{1}{c}{ Frecuencia } & \multicolumn{1}{c}{$\%$} \\
\hline Muy buena & 342 & $71 \%$ \\
Regular & 134 & $28 \%$ \\
Mala & 5 & $1 \%$ \\
Total & 481 & $100 \%$ \\
\hline
\end{tabular}

Fuente: Datos primarios de la encuesta. Junio 2015

Según los datos obtenidos en elCuadro $\mathrm{N}^{\circ} 12$; la mayoría de los clientes representados por el $73 \%$ adquieren estos productos solo cuando realizan construcción o si deciden realizar reparaciones en sus hogares, en cambio un 21\% mencionó comprar de manera mensual en las empresas debido a que son albañiles e ingenieros y los utilizan constantemente para realizar construcciones o remodelaciones en los hogares de sus jefes. En cambio el $2 \%$ reflejó realizar sus compras quincenalmente ya que ejecutan arreglos menores en sus hogares o por que trabajan en proyectos.
Cuadro $N^{\circ} 12$ Frecuencia con que realiza compras

\begin{tabular}{lll}
\hline \multicolumn{3}{c}{ Universo de estudio 481 encuestas } \\
\hline \multicolumn{1}{c}{ Frecuencia } & Frecuencia & \% \\
\hline Obras de construcción & 351 & $73 \%$ \\
Mensual & 100 & $21 \%$ \\
Quincenal & 12 & $2 \%$ \\
Otros & 18 & $4 \%$ \\
Total & 481 & $100 \%$ \\
\hline
\end{tabular}

Fuente: Datos primarios de la encuesta. Junio 2015

El $100 \%$ de los encuestados coinciden que las principales ventajas que brinda la ubicación de las empresas comercializadoras de materiales de construcción es la accesibilidad ya que facilita la transportación de los materiales adquiridos. Algunos de los clientes comentaron que para ellos es más económico que las empresas se encuentren ubicadas a orillas de la carretera panamericana y en el centro, que en lugares alejados, ya que traería consigo gastos adicionales, en otros casos dijeron que las empresas les brindan área de parqueo con un $40 \%$ esto representa un beneficio para los clientes que realizan las compras en sus vehículos o que llegan a retirarlos porque lo pueden hacer de una manera más cómoda y sin afectar el tráfico.

Cuadro $N^{\circ} 13$ Ventajas de la ubicación

\begin{tabular}{lll}
\hline \multicolumn{3}{c}{ Universo de estudio 481 encuestas } \\
\hline \multicolumn{1}{c}{ Ventajas } & Frecuencia & $\%$ \\
\hline Fácil acceso & 481 & $100 \%$ \\
Facilidad para transportar & 320 & $67 \%$ \\
los materiales & & \\
Área de parqueo & 80 & $40 \%$ \\
\hline
\end{tabular}

Fuente: Datos primarios de la encuesta. Junio 2015

Según datos reflejados en el cuadro $\mathrm{N}^{\circ} 14$; obtenidos mediante el estudio la mayor parte de los encuestados reflejaron que les han brindado asesoría a cerca de los materiales que van a adquirir lo que les ha permitido tener mayores conocimientos por lo cual el $83 \%$ aducen que los asesores están bien capacitados para desempeñar esta función. Un 17\% afirmaron que algunos asesores deben recibir mejores capacitaciones 
o adquirir mayores conocimientos sobre estos y así brindar un servicio de mayor calidad.

\begin{tabular}{|c|c|c|}
\hline \multicolumn{3}{|c|}{ Universo de estudio 481 encuestas } \\
\hline Capacidad de venta & Frecuencia & $\%$ \\
\hline$\overline{\mathrm{Si}}$ & 399 & $83 \%$ \\
\hline No & 82 & $17 \%$ \\
\hline Total & 481 & $100 \%$ \\
\hline
\end{tabular}

Fuente: Datos primarios de la encuesta. Junio 2015

Se determinó que tan satisfechos se encuentran los clientes de la asesoría brindada; el $82 \%$ considera que la asesoría es muy buena, debido a que les atienden con amabilidad y les han dado sugerencias de que materiales son los más adecuados para su construcción o cualquier remodelación, mientras tanto un $2 \%$ de los clientes valoran como mala la asesoría brindada debido a que estos no son atendidos con rapidez y en ocasiones las personas carecen de amabilidad y de paciencia.

Cuadro $N^{\circ} 15$ Valoración de la asesoría

\begin{tabular}{lll}
\hline \multicolumn{3}{c}{ Universo de estudio 481 encuestas } \\
\hline \multicolumn{1}{c}{ Valoración } & Frecuencia & \multicolumn{1}{c}{$\%$} \\
\hline Muy Buena & 395 & $82 \%$ \\
Regular & 76 & $16 \%$ \\
Mala & 10 & $2 \%$ \\
Total & 481 & $100 \%$ \\
\hline
\end{tabular}

Fuente: Datos primarios de la encuesta. Junio 2015

Para decidir en qué empresa realizar las compras los clientes toman en cuenta los beneficios que esta les puedan ofrecer. Obteniendo que el $67 \%$ de las personas que compra una cierta cantidad de materiales, reciben como principal beneficio los descuentos, permitiéndoles realizar más compras. Por otra parte el 37\% de las personas encuestadas aducen no haber recibido ningún benefició por parte de las empresas debido a que tienen poco tiempo de realizar sus compras en esta o hasta ahora las están realizando. Posteriormente en el cuadro $\mathrm{N}^{\circ} 17$, se consultó si realmente las empresa cumplen con los beneficios que les ofrecen, determinando que el $60 \%$ de los clientes consideran que si cumplen en tiempo y forma con los beneficios ofrecidos.
Cuadro $N^{\circ} 16$ Beneficios ofrecido por los negocio

\begin{tabular}{lcc}
\hline \multicolumn{3}{c}{ Universo de estudio 481 encuestas } \\
\hline \multicolumn{1}{c}{ Capacidad de venta } & Frecuencia & $\%$ \\
\hline Entrega a domicilió & 103 & $21 \%$ \\
Promociones & 181 & $38 \%$ \\
Descuesto & 325 & $67 \%$ \\
Otros (Especifique) & 104 & $22 \%$ \\
Ninguno & 178 & $37 \%$ \\
\hline
\end{tabular}

Fuente: Datos primarios de la encuesta. Junio 2015

Cuadro $N^{\circ} 17$ Cumplen con los beneficios.

\begin{tabular}{lccc}
\hline \multicolumn{3}{c}{ Universo de estudio 481 encuestas } \\
\hline & Cumplen & Frecuencia & \% \\
\hline $\mathrm{Si}$ & 288 & $60 \%$ \\
$\mathrm{No}$ & 193 & $40 \%$ \\
Total & 481 & $100 \%$ \\
\hline
\end{tabular}

Fuente: Datos primarios de la encuesta. Junio 2015

Según los datos obtenidos el 73\% del universo de los clientes consideran sentirse satisfechos por las compras que han realizado en dichas empresas ya que en la mayoría han obtenido productos de calidad y han sido muy bien atendidos por los vendedores quienes además les dan a conocer productos nuevos que han salido al mercado y les explican cómo utilizarlos, a su vez dieron a conocer de algunas capacitaciones que les brindan estas empresas por ser clientes permanentes en el caso de ingenieros, albañiles o maestros de obras. No obstante el otro $27 \%$ dice no sentirse totalmente satisfechos ya que en ocasiones les han dado un material por otro o les han vendido un producto que no posee la medida exacta a como ellos se lo han solicitado.

Cuadro $N^{\circ} 18$ Satisfacción por las compras Universo de estudio 481 encuestados

\begin{tabular}{lccc}
\hline \multicolumn{3}{c}{ Cuadro $\mathbf{N}^{\circ}$ 18 Satisfacción por las compras } \\
\hline & Universo de estudio 481 encuestados \\
\hline $\mathrm{Si}$ & Satisfacción & Frecuencia & $\%$ \\
$\mathrm{No}$ & 350 & $73 \%$ \\
Total & 131 & $27 \%$ \\
\hline
\end{tabular}

Fuente: Datos primarios de la encuesta. Junio 2015 
Según el 56\% de los encuestados, las empresas donde ellos realizan sus compras les ofrecen precios accesibles en comparación a los demás negocios, y en muchas ocasiones les brindan algún tipo de incentivo, lo cual motiva sus compras, no obstante aseguran que para ellos es mejor que los productos sean baratos ya que les permite adquirir más materiales y ahorrar dinero, en cambio un $44 \%$ de los encuestados aducen que los precios suelen ser algo altos pero que los productos que ofrecen poseen grandes estándares de calidad y duran más que otros, además a la larga les ahorran dinero y poseen mayor durabilidad que los de la competencia.

\begin{tabular}{|c|c|c|}
\hline \multicolumn{3}{|c|}{ Cuadro $N^{\circ} 19$ Accesibilidad de los precios brindad } \\
\hline \multicolumn{3}{|c|}{ Universo de estudio 481 encuestados } \\
\hline Precios & Frecuencia & $\%$ \\
\hline $\mathrm{Si}$ & 271 & $56 \%$ \\
\hline No & 210 & $44 \%$ \\
\hline Total & 481 & $100 \%$ \\
\hline
\end{tabular}

Fuente: Datos primarios de la encuesta. Junio 2015

Para la mayoría de los clientes los incentivos son importantes debido a que influyen en la elección de la empresa donde desean realizar sus compras. Un 56\% afirma obtener algún tipo de incentivo por parte de las empresas, no obstante el otro $44 \%$ dice no obtener ningún tipo de incentivo, sin embargo consideran que dichos negocios cuentan con grandes estándares de calidad por lo que no es necesario para ellos que brinden incentivos ya que de igual manera adquirirán sus productos.

Cuadro N$^{\circ} 20$ Le brindan algún tipo de incentivo

\begin{tabular}{lccc}
\hline \multicolumn{3}{c}{ Universo de estudio 481 encuestados } \\
\hline & Incentivos & Frecuencia & \% \\
\hline $\mathrm{Si}$ & 270 & $56 \%$ \\
$\mathrm{No}$ & 211 & $44 \%$ \\
$\mathrm{Total}$ & 481 & $100 \%$ \\
\hline
\end{tabular}

Fuente: Datos primarios de la encuesta. Junio 2015

Según la información obtenida anteriormente la mayoría de los clientes dicen recibir algún tipo de incentivo, por lo que se decidió indagar sobre cuáles son los incentivos que reciben. A lo que reflejaron con un $100 \%$ que los descuentos sobre compras son los que más reciben por parte de las empresas, sin embargo algunos clientes dicen obtener entre $3 \%$ a $5 \%$ de descuentos, por otro lado un $81 \%$ dice obtener créditos por parte de las empresas, lo que se debe a que en su mayoría son clientes que llevan años de adquirir sus productos en estos negocios. En cambio un $37.4 \%$ de la muestra de estudio dicen obtener otros incentivos como las regalías, es decir por un producto se da el otro más barato o se le regala.

Cuadro $\mathrm{N}^{\circ} 21$ Le brindan algún tipo de incentivo

\begin{tabular}{lll}
\hline \multicolumn{3}{c}{ Universo de estudio 481 encuestados } \\
\hline \multicolumn{1}{c}{ Incentivos } & Frecuencia & \% \\
\hline Promociones & 298 & $62 \%$ \\
Crédito & 390 & $81 \%$ \\
Descuentos & 481 & $100 \%$ \\
Pagos por cuota & 320 & $66.5 \%$ \\
Otro & 180 & $37.4 \%$ \\
\hline
\end{tabular}

Fuente: Datos primarios de la encuesta. Junio 2015

Para los clientes es importante que la empresa donde realizan sus compras les oferten todo lo que necesitan por lo que se consideró necesario saber si ellos consideran que la empresa vende lo que ellos demandan, con un $64 \%$ es decir la mayoría de ellos dicen encontrar todo lo que ellos, ya que la empresa vende gran variedad de productos, marcas y estilos. No obstante hay quienes dicen no encontrar todo lo que ellos andan buscando ya que la empresa no cuenta con gran variedad de producto y el local es pequeño para incluir más productos.

Cuadro $\mathrm{N}^{\circ} 22$ Encuentra todo lo que necesita

\begin{tabular}{|c|c|c|}
\hline \multicolumn{3}{|c|}{ Universo de estudio 481 encuestado } \\
\hline Variedad & Frecuencia & $\%$ \\
\hline $\mathrm{Si}$ & 310 & $64 \%$ \\
\hline No & 171 & $36 \%$ \\
\hline Total & 481 & $100 \%$ \\
\hline
\end{tabular}

Fuente: Datos primarios de la encuesta. Junio 2015 


\section{Propuesta de estrategia de comercialización:}

\begin{tabular}{|c|c|}
\hline FORTALEZAS & DEBILIDADES \\
\hline $\begin{array}{l}\text { - Posicionamiento en el mercado. } \\
\text { - Buen servicio al cliente. } \\
\text { - Experiencia en la venta de materiales de construcción. } \\
\text { - Variedad de productos. } \\
\text { - Ubicación que permita una mayor accesibilidad al } \\
\text { cliente. }\end{array}$ & $\begin{array}{l}\text { - Falta de transporte propio para realizar entregas de } \\
\text { pedidos. } \\
\text { - } \quad \text { No se cuenta con asesores capacitados. } \\
\text { - } \quad \text { Falta de organización para entrega de pedidos. } \\
\text { - Poco uso de la publicidad. }\end{array}$ \\
\hline OPORTUNIDADES & AMENZAS \\
\hline $\begin{array}{l}\text { - } \text { Auge del sector de construcción en el norte del País. } \\
\text { - } \quad \text { Crecimiento económico de la ciudad. } \\
\text { - } \quad \text { Opjores condiciones de mercado. } \\
\text { - } \quad \text { Mayil como son las capacitaciones. } \\
\text { - }\end{array}$ & $\begin{array}{l}\text { - Gran cantidad de competidores actuales. } \\
\text { Ingreso de Nuevos competidores nacionales e } \\
\text { internacionales. } \\
\text { - Los precios bajos de la competencia. }\end{array}$ \\
\hline
\end{tabular}

En base al FODA se prosiguió a realizar un análisis FODA el cual permitió diseñar estrategias que permitan reducir las debilidades y potenciar las fortalezas y oportunidades que logren conducir al desarrollo de las diferentes empresas competidoras de materiales de construcción.

\section{Estrategia 1: Sondeos de opinión.}

Objetivo: Implementar sondeos de opinión para conocer el grado de aceptación de los productos por parte de los clientes.

\section{Estrategia 2: Diversificación de Producto.}

Objetivo: Disponer de una variada gama de materiales que genere una mayor ventaja competitiva.

\section{Estrategia 3: Capacitación al Personal.}

Objetivo: Efectuar capacitaciones que permitan al personal adquirir mayores conocimientos sobre los productos y atención al cliente.

\section{Estrategia 4: Uso de Publicidad.}

Objetivo: Utilizar frecuentemente medios de publicidad para llegar a más sectores de la ciudad de Estelí y municipios aledaños.
Estrategia 5: Valor agregado (Entregas a Domicilio) Objetivo: Brindar un mejor servicio cumpliendo en tiempo y forma con la entrega de los pedidos.

Según la información recopilada por medio de la investigación y el análisis realizado a las empresas comercializadoras de materiales de construcción se puede concluir que:

- Las principales estrategias competitivas utilizadas por las empresas comercializadoras de materiales de construcción de la ciudad de Estelí son: buena atención al cliente, implementación de incentivos, diversificación de materiales $y$ beneficios adicionales los cuales permiten obtener una mejor captación de clientes y mantener a los que ya poseen.

- Según se refleja en el cuadro N³ se puede decir que la hipótesis de la investigación se cumple con un porcentaje del $67 \%$, lo cual demuestra que la diversificación de materiales es una de las fortaleza que les ha permitido tener éxito a los propietarios o encargados de las empresas, además consideran que la atención influyen en la satisfacción de los clientes, lo que se demuestra con un porcentaje del $90 \%$; de igual forma los clientes opinan que lo que buscan en las empresas es la diversificación 
de materiales a como muestra el cuadro $\mathrm{N}^{\circ} 10$ con el porcentaje del $58 \%$, porque les permite ahorrar tiempo y dinero al adquirir todo los materiales que necesitan en una misma empresa.

- La ubicación es un aspecto que les ha permitido posicionarse y mantenerse en el mercado ya que la mayoría de las empresas encuestadas se encuentran en los distritos I y II o sea a orillas de la carretera panamericana y el centro de la ciudad lo que les permite a los clientes mayor accesibilidad y facilidad para transportar sus adquisiciones.

- Por medio del análisis de las entrevistas y encuestas aplicadas se puede decir que la publicidad ya sea radial, televisiva, pancartas o por volantes influye en la captación de clientes ya que es a través de esta que se le brinda información de la ubicación de las empresas, de sus materiales o de las promociones que estén brindando.

\section{RECOMENDACIONES}

Afín de que la presente investigación sea de gran utilidad para las empresas comercializadoras de materiales de construcción se les recomienda:

- Realizar capacitación al personal de venta para que brinden una mejor asesoría a los clientes, lo que permitirá tener un mayor grado de captación y preferencia.

- Implementar nuevas estrategias como la comercialización de materiales modernos ya que a través de estos las empresas lograran exclusividad.

- Realizar seguimiento a sus clientes permanentes ya sea a través de visitas, correo electrónico o llamadas telefónicas.

- Siendo la satisfacción al cliente el principal factor de importancia para estas empresas se recomienda establecer buzón de sugerencias en los establecimientos para conocer el nivel de satisfacción que están brindando.

- Utilizar medios de publicidad, ya sean radiales, televisivos u otros que den a conocer a la empresa para obtener consigo mayor afluencia de clientes.

\section{BIBLIOGRAFÍA}

Gómez, M. (2005). Competitividad. Recuperado el 18 de 04 de 2015, de Definición de Competitividad: http://www.zonaeconomica.com/definicion/ competitividad

Groover, M. P. (2002). Variedad de Productos. Fundamentos de manufactura moderna. Materiales, procesos y sistemas 1a. Ed. México: Pearson. Prentice Hall.

Kotler, P. (2008). Satisfacción del cliente. México: Pearson. Educación. 